\title{
Le programme de recherches du Centre National pour l'Exploitation des Océans sur l'aménagement du littoral
}

\author{
par R. Toussaint \\ Chargé de l'aménagement du littoral à la Direction \\ des Programmes du C.N.E.X.O.
}

Le C.N.E.X.O., organisme para-public, créé en 1967, a une vocation nationale de coordination des recherches océanographiques visant à exploiter les océans. De plus, il a pour mission de conseiller les pouvoirs publics et d'inciter à la promotion d'idées et de techniques nouvelles liées à l'exploitation des océans. Cette mission générale concerne l'exploitation des ressources vivantes (pêche et aquaculture), des agrégats marins (sables et graviers), le développement des techniques d'intervention de l'homme dans le milieu marin, la lutte contre les pollutions, l'interaction entre l'océan et l'atmosphère. Les recherches sur l'aménagement du littoral ont une vocation géographique et concourent aux missions générales du C.N.E.X.O.

Le littoral est une aire géographique composée d'une bande terrestre longeant le trait de côte, associée à une frange marine adjacente; les limites de ces deux entités complémentaires peuvent être définies en fonction de leurs interactions et des activités spécifiques qui y prennent naissance.

Seule, la frange marine du littoral constitue le domaine d'application du programme de recherches du C.N.E.X.O., concerné ici. Dans certains cas, il s'agira de l'ensemble du plateau continental.

D'une façon générale, cette frange marine renferme, dans son sol et son sous-sol, de nombreuses ressources minérales et fossiles. D'autre part, c'est une zone privilégiée de contacts et d'échanges entre le fond, le milieu liquide et l'atmosphère qui favorisent le développement de la vie marine. Ces échanges sont particulièrement importants dans les zones proches des côtes notamment dans les estuaires, lagunes, marais et étangs littoraux où les apports d'eau douce et de sels minéraux terrigènes accroissent la production primaire et done la production halieuthique.

Pour la plupart des responsables de l'aménagement, le littoral n'a été jusqu'à présent qu'une bande de terre dont l'originalité était d'être adossée à la mer; une frange relativement étroite, aux contours assez flous dont les limites ne dépassaient guère celles de la plage et de ses accès immédiats, celles du port, de la station balnéaire.

Depuis peu, on se rend compte que cette notion est trop restrictive. Les aménageurs ne peuvent plus ignorer la nature du milieu marin, l'exploitation de ses ressources actuelles et potentielles. Toutes ces activités maritimes doivent être organisées, surtout dans les zones côtières là où se concentrent la plupart d'entre elles. "Aménager la mer »; l'idée est lancée; reste à savoir ce qu'elle peut signifier et ce qu'on peut en attendre.

L'aménagement, c'est l'action qui consiste à répartir, dans un cadre géographique, des activités économiques, en fonction des ressources naturelles et humaines. La mer, en tant que cadre géographique, peut parfaitement se prêter à cette définition.

Créer des cantonnements, draguer des chenaux, poser des câbles sous-marins, autoriser des dépôts en mer, prélever des agrégats, exploiter du pétrole off shore, fixer des frontières juridiques, concéder des terrains sur le domaine public maritime sont, par exemple, des actions d'aménagement de la mer.

Le littoral est une zone particulièrement convoitée, à vocations multiples, où se focalisent des activités économiques dont l'occupation engendre des conflits; bref, c'est un bien rare qu'il faut savoir sauvegarder, préserver, mais aussi équiper et exploiter.

Le littoral est un lieu de convergence du tourisme. Il tend à perdre son caractère de fréquentation saisonnière pour devenir, en plus, un site d'habitat permanent. En temps que carrefour terre-mer, le littoral est une zone de choix pour l'implantation d'industries de transformation et d'un important secteur tertiaire lié au transit des marchandises et des personnes. Quant à la frange marine, elle renferme de nombreuses ressources exploitées et exploi- 
tables d'un apport économique croissant: l'aquaculture, les exploitations d'agrégats, par exemple.

Les vocations multiples de la zone littorale expliquent les conflits qui prennent naissance quant à son occupation et à l'affectation de ses sites. Ces conflits mettent souvent en évidence des incompatibilités de voisinage entre tel ou tel secteur d'activité.

L'essor des activités touristiques, le développement d'implantations industrielles, de zones portuaires, de structures urbaines, de grands équipements tels que centrales électriques, le développement de l'exploitation des océans et particulièrement du plateau continental, la nécessité de sauvegarder la nature et un cadre de vie agréable, conduisent déjà et conduiront davantage l'autorité politique à prononcer des choix.

Entre le développement du ski nautique et la culture des huîtres, entre l'exploitation d'agrégats en mer et le risque de destruction ou de déplacement d'espèces vivantes, entre les exploitations de pétrole off shore et les risques y afférants, entre la construction d'un port de plaisance, d'une marina et la sauvegarde d'un site naturel, entre l'implantation d'une raffinerie et les risques de nuisances sur les parcs conchylicoles, etc., quels sont les éléments des choix, quels sont les critères scientifiques, techniques, économiques et humains qu'il faut mettre en valeur pour aider l'autorité politique à choisir en connaissance de cause?

Tel est le cadre dans lequel le C.N.E.X.O. agit en liaison étroite avec les organismes et administrations concernés.

\section{La mission}

La mission du C.N.E.X.O. en matière d'aménagement du littoral se résume de la façon suivante:

- Conseiller, sur le plan scientifique et technique, les Pouvoirs Publics et les collectivités responsables quant aux projets d'aménagements (équipements, exploitations, moyens de sauvegarde du milieu) pouvant comporter des incidences sur le milieu marin et les activités.

- Inciter et promouvoir des idées nouvelles visant l'aménagement de la frange marine du littoral au niveau:

- des conceptions techniques d'équipements;

- des moyens d'une politique de gestion des ressources marines et des activités;

- des possibilités de développement des activités liées à la mer et ses ressources et leur insertion géographique, économique et humaine.

\section{Les objectifs} tifs :

Cette mission se traduit par quatre ensembles d'objec-

a) Acquérir une meilleure connaissance du milieu marin littoral quant à sa nature, son évolution, ses ressources, ses utilisations; b) Mettre en évidence les tendances des utilisations, les conflits possibles, les aptitudes des sites vis-à-vis des activités;

c) Inciter au développement de techniques nouvelles d'exploitation et d'équipement;

d) Définir des modèles de gestion des ressources naturelles et des activités pour aboutir à des critères de choix d'aménagement.

\section{Le programme}

D'une façon pratique, et pour atteindre ces objectifs généraux, les recherches, dont certaines ont d'ores et déjà été engagées, sont définies suivant quatre rubriques fondamentales:

1 - Les inventaires et analyses de données;

2 - Les recherches, méthodes et modèles;

3 - Les études locales;

4 - Les technologies.

D'une façon résumée, ces rubriques comportent les études suivantes :

\section{1 - Les inventaires ef analyses de données}

De multiples études sectorielles (pêche, conchyliculture, hydrologie, biologie, sédimentologie, par exemple) et locales ont été menées sur le littoral. Un effort d'inventaire, de synthèse, de compréhension, de corrélation reste à faire pour tirer le meilleur des études actuelles. A cet effet, un système de documentation automatique est en cours de mise en place au B.N.D.O. pour l'aménagement du littoral. Des synthèses régionales de données scientifiques et économiques sont aussi en cours et se poursuivront pendant plusieurs années pour aboutir à une harmonisation de la collecte et du traitement des données.

\section{2 - Les recherches, méthodes ef modèles}

Ces inventaires ne peuvent être utilisables que dans la mesure où ils sont doublés par des recherches sur la signification des paramètres (houles, courants, espèces vivantes, nature des fonds, par exemple), leurs utilisations pratiques à des fins d'aménagement et par des recherches méthodologiques sur des modèles prévisionnels de gestion. Dans ce dernier domaine, un effort très important reste à faire sur le plan national. La modélisation des effets de la houle sur le fond, des transports de sédiments dans les estuaires, de l'écologie en milieu estuairien sont des exemples de recherches prévues au titre de ce programme. L'ensemble de ces recherches devrait aboutir à l'expression de modèles de gestion sous forme par exemple de Schéma d'Aptitudes et d'Utilisations de la Mer (S.D.A.U.M.).

\section{3 - Les études locales}

Chaque projet d'équipement ou d'exploitation nécessite des études particulières malgré les recherches générales 
menées par ailleurs dans ce programme. Il s'agit, dans cette rubrique, des applications concrètes sur des projets réels. La confrontation de nombreuses études locales orientées par leur caractère concret, et des recherches générales, permettra de juger des limites d'application des méthodes et modèles pour aboutir à la notion de critères de choix et d' "arguments significatifs » vis-à-vis des responsables de l'aménagement du littoral.

\section{4 - Les technologies}

L'aménagement est lié en grande partie au développement technologique. Des techniques nouvelles de structures en off shore permettraient de proposer des variantes à des projets d'équipements (centrales nucléaires, par exemple) et de faciliter le développement de nombreuses activités (tourisme, industrie, exploitations, etc.).

L'utilisation de telles techniques nécessite des études de faisabilité et d'incidences qui font l'objet de cette rubrique du programme.

\section{Conclusion}

La présence de la mer, avec ses ressources et ses utilisations, justifie les caractéristiques propres du littoral. De ce fait, la notion de littoral doit comprendre l'ensemble du plateau continental.

Si l'homme peut vivre sur le littoral le dos tourné à la mer, le responsable de l'aménagement ne peut plus ignorer le milieu marin et toutes les possibilités qu'il offre, aussi bien dans le domaine des activités maritimes traditionnelles que nouvelles. Pour ses projets d'équipements, il doit aussi penser aux solutions originales que peut lui offrir le développement de techniques nouvelles telles que les constructions off shore. Mais, il devra aussi être prudent, car les écosystèmes marins sont fragiles et précaires. L'environnement maritime doit être préservé et conservé en tant que capital de richesses naturelles, indispensable à la vie sur la terre.

Pour arriver à un développement harmonieux de l'aménagement du plateau continental en tant que partie intégrante du littoral, il est indispensable de continuer de nombreuses recherches et études sur la connaissance du milieu marin et la compréhension des mécanismes qui le régissent, ainsi que sur la gestion rationnelle de ses ressources et des activités.

L'horizon de l'aménageur ne doit plus s'arrêter à l'écume des vagues qui se brisent sur les rivages. Il doit voir plus loin et tenir compte de l'exploitation du plateau continental et de ses développements possibles.

Ceux-ci peuvent avoir des incidences directes sur l'aménagement et l'utilisation de sites purement côtiers. Il s'agit, par exemple, de l'aquaculture dont le développement industriel nécessitera tout un ensemble d'espaces favorables qu’il est nécessaire de "réserver » d’ores et déjà.
Les exploitations d'agrégats marins modifieront les habitudes des industriels concernés, les circuits commerciaux, et nécessiteront le développement d'infrastructures particulières dans les zones portuaires. Ces exploitations ne manqueront pas de provoquer quelques inquiétudes de la part des pêcheurs. Des choix économiques seront à faire et les incidences éventuelles de ces exploitations devront être étudiées avec précision, en dissociant les effets temporaires des effets définitifs.

Enfin tout en favorisant l'exploitation du plateau continental pour le bien-être de tous, "l'aménageur de la mer" devra contrôler et organiser son développement en fonction des besoins économiques et humains, des compatibilités et des incompatibilités d'usages. Un outil de gestion leur manque: les "Schémas d'Aptitudes et d'Utilisations de la Mer» (S.D.A.U.M.).

Un tel outil permettrait, au moins dans un premier temps de :

- représenter la nature des usages du milieu marin;

- déterminer les aptitudes intrinsèques des sites maritimes et les contraintes correspondantes;

- préciser les usages compatibles avec les aptitudes;

- fixer les droits attachables aux sites maritimes quant à leurs usages et leurs limites dans le temps et l'espace.

La conception, les finalités précises et les modalités d'exécution de cet outil restent à définir. Le dernier point, notamment, est encore à évoquer avec de multiples réserves. Il devrait soulever, dans l'avenir, de nombreux débats en raison du caractère inaliénable de la mer, de certaines dispositions réglementaires qui accordent des privilèges à des catégories professionnelles, de l'évolution des techniques d'exploitation des richesses marines qui laissent entrevoir des horizons nouveaux et de tous les aspects sociaux, politiques et économiques qui s'y greffent. Néanmoins, un S.D.A.U.M. permettrait :

- de donner une information multisectorielle aux utilisateurs:

- d'orienter l'aménagement du littoral en tenant compte des usages sur sa frange marine et, ce fait, créer un instrument de concertation;

- de définir et mettre en cuvre une politique de sauvegarde et de protection du milieu marin;

- de ménager des espaces favorables à des usages spécifiques liés à la mer et de créer des outils promotionnels pouvant favoriser le développement concerté de certaines activités (image de marque dominante);

- d'infléchir les investissements publics et privés et l'exploitation des ressources naturelles, afin de pourvoir à une mise en valeur rationnelle et harmonieuse du milieu marin dans le contexte des développements économiques et humains régionaux ou locaux.

Mais tout ceci ne serait que vains mots et idées creuses si les Pouvoirs Publics, les responsables de l'aménagement du littoral, les exploitants du milieu marin ne se sentent pas concernés directement et ne décident pas, ensemble, de se donner les moyens de gérer rationnellement la frange marine du littoral. 


\section{Discussion}

Président : M. A. BRIN

M. le Président remercie $M$. Toussaint de son intéressant exposé et ouvre la discussion.

Vous avez évoqué, dit $M$. BANAL, le problème de la fermeture des estuaires. C'est, en effet, une question très importante car une des façons de résoudre le problème des estuaires est de les fermer purement et simplement. C'est d'ailleurs, dans une certaine mesure, ce qui a été fait en Hollande et il y a là un exemple tout à fait remarquable.

Le problème de l'environnement se réduit, pour certains, à conserver ce qui existe. Mais les estuaires, sous leur aspect actuel, résultent peut-être d'une circonstance assez fortuite : une évolution ancienne du nivcau de la mer; les choses pourraient fort bien être différentes et, en particulier, il pourrait ne pas y avoir d'estuaires; on peut donc les supprimer sans commettre un crime de "lèseenvironnement 》.

\section{Toussaint répond:}

Je vais faire une réponse de Normand en disant : que se passeraitil si tous les estuaires français étaient barrés? Pour quelques scientifiques, barrer certains estuaires ne fait qu'accélérer un processus naturel. Beaucoup d'entre eux, notamment en raison de prélèvements d'eau en amont et de transits sédimentaires, se bouchent d'eux-mêmes. Sur le plan écologique, il n'en résulterait peut-être pas de gros risques; pourtant on ne dispose d'aucun critère permettant de l'affirmer.

De toute façon, il est reconnu que les estuaires sont d'un intérêt capital pour l'écologie marine côtière. L'essentiel est de connaître les limites à imposer dans les processus d'aménagement des estuaires. Pour l'instant, on ne dispose d'aucun critère valable. Le C.N.E.X.O. se propose de rechercher de tels critères.

Le Professeur PÉRÈs pourra, tout à l'heure, donner quelques précisions sur ce point. Le C.N.E.X.O. se pose simplement la question et ne cherche pas, dans un premier temps, à la résoudre. Sans doute, certains équipements ont peu d'infuence sur l'enyironnement mais, actuellement, nous n'avons que des critères extrêmement subjectifs pour en juger.

Les estuaires sont-ils des endroits privilégiés pour les établissements d'aquaculture; quel est le régime administratif de tels établissements, demande M. RÉméní́ras?

Je pense, tépond $M$. Toussaint, que les estuaires sont très favorables à certaines formes d'aquaculture qui nécessitent des apports d'eau douce; il en est ainsi, par exemple, pour l'élevage des salmonidés. Les estuaires sont aussi bien adaptés à la conchyliculture et à la mytiliculture, surtout à la conchyliculture.

Le problème de l'occupation de la mer est extrêmement intéressant. La mer est inaliénable et constitue un domaine public. Au cours des dernières décennies, les choses et l'exploitation de la mer, en particulier, ont considérablement évolué. Néanmoins, le fait de concéder des terrains sur le domaine public maritime - de créer des parcs conchylicoles, par exemple - conduit à une certaine forme d'occupation; il est assez normal qu'une personne morale, qui investit pour améliorer ces terrains parce qu'elle en a la concession, ait une certaine propriété de ces terrains.

Le problème va se poser dans les mêmes termes en matière d'aquaculture, non seulement sur le domaine public maritime, mais aussi un peu plus au large, et les diférentes catégories socio-professionnelles intéressées - notamment les marins pêcheurs, les anciens inscrits maritimes - estiment avoir un droit dans ce domaine.

Pour les grands estuaires, comment concevez-yous la collaboration entre les organismes chargés de leur aménagement, les Ports Autonomes par exemple, et le C.N.E.X.O.? demande M. LESPINE.

M. Toussant répond en ces termes :

Dans le cas oì il existe des organismes responsables de l'aménamement des grands estuaires dotés de moyens importants, il n'y a aucune raison pour que le C.N.E.X.O. apporte systématiquement des moyens supplémentaires. Néanmoins, le C.N.E.X.O., en tant qu'organisme public national est intéressé à certaines études et recherches, à condition qu'elles aient un caractère général et extrapolable. Or, ce qui est le plus intéressant à mon sens, ce sont les estuaires qui n'ont pas la chance d'avoir des organismes puissants responsables, capables de mener les études nécessaires et de prévoir les incidences de leur équipement. Mais ce sont surtout les petits estuaires qui retiennent pour l'instant mon attention, car les études y sont beaucoup moins nombreuses. Pour les grands estuaires, qui possèdent des ports autonomes, le C.N.E.X.O. peut intervenir pour aider le développement de certaines recherches, à condition qu'elles aient un caractère général. Autrement dit, ce n'est pas la mission du C.N.E.X.O. de s'immiscer dans des problèmes locaux qui sont du ressort des ports autonomes, par exemple. C'est très simple en théorie, peut-être moins dans la pratique.

Ayant travaillé pour le compte du Port Autonome de Nantes en tant que membre de la Faculté des Sciences de Rennes et de Brest, observe M. Mauvars, je pense, maintenant que je travaille au C.N.E.X.O, gue cet organisme, disposant d'un ordinateur assez puissant, pourrait aider les Ports Autonomes qui feraient appel à lui pour certains problèmes de calcul en particulier.

Avez-vous pu chiffrer le coût du programme général que vous avez exposé ? demande M. Gibrat.

Vous posez là une question à laquelle je suis incapable de répondre précisément, dit $M$. Toussanx. Faire le point des décennies d'études et de recherches effectuées jusqu'à ce jour sur les côtes de France, je l'évalue personnellement, d'une façon très grossière, et par extrapolation sur nos régions, à une dépense de lordre de 500 millions d'anciens francs. Ce n'est peut-être pas considérable et on peut répartir la dépense sur plusieurs années.

M. BANAL intervient en ces termes :

II y a plusieurs catégories d'estuaires. Les trois grands dont vous avez parlé et que je connais bien, n'en parlons pas, puisque vous avez dit qu'ils étaient un peu en dehors de vos préoccupations immédiates. Ensuite, il y a ce que j'appelle les estuaires moyens, dont le type est celui de la Somme; il y en a probablement une dizaine en France. Enfin, il y a les tout petits, dont l'étude n'est pas distincte de celle de la côte proprement dite. C'est finalement, semble-t-il, sur les estuaires moyens que se pose un problème spécifique, qui n'est pas actuellement l'objet d'un traitement particulier par un Service véritablement concemé (encore que le Service de l'Equipement de la Somme serait probablement assez vexé s'il m'entendait). Est-ce que ces estuaires vont tenir, dans votre programme, une place appréciable ou très marginale? Considérezvous que l'étude de ces estuaires moyens est peu de chose par rapport à l'ensemble de la France ?

Je voudrais d'abord rappeler, répond $M$. Toussans, que notre programme sur l'aménagement du littoral a commencé à être mis à l'étude au C.N.E.X.O. depuis un an; donc il n'a pas cncore atteint sa maturité. D'autre part, nous avons, dès le départ, voulu lui donner ce caractère régional que j'ai évoqué tout à l'heure. Pour diverses raisons, la conjoncture nous a fait choisir la Bretagne. C'est dans ce contexte régional que nous sommes amenés à étudier les problèmes spécifiques des estuaires, de façon à en prévoir les aménagements dans le cadre de schémas tenant compte des contraintes grossières dues au milieu marin et aux activités économiques maritimes. Nous n'avons pas encore commencé l'étude systématique des estuaires, sauf pour la Bretagne; nous allons continuer l'an prochain en vue de la réalisation de la «fiche technique $\diamond$ de ces estuaires bretons, de façon à mieux les comprendre et à mieux préciser, vís-à-vis des responsables de l'aménagement, les contraintes qu'ils imposent. Mais nous n'avons pas encore commencé l'étude systématique des estuaires.

M. le Président clôt la discussion en remerciant $M$ Toussaint et tous ses interlocuteurs et donne la parole à $\mathrm{M}$. le Professeur Pérès, membre de l'Académie des Sciences, pour l'exposé de sa communication. 